\title{
Biodiversité des emballages-feuilles végétales utilisées dans l'artisanat agroalimentaire au Sud du Bénin
}

\author{
ONZO F.Caroline ${ }^{1,}$ AZOKPOTA Paulin ${ }^{* 1}$, AKISSOE Noël2 et AGBANI O.Pierre ${ }^{3}$ \\ 1 Laboratoire de Formulations Alimentaires et de Biologie Moléculaire (LAFAB) ; Département de Nutrition, \\ Sciences et Technologie Alimentaires, Faculté des Sciences Agronomiques, Université d'Abomey-Calavi, 01 BP \\ 526, Cotonou, Bénin ; \\ 2 : Laboratoire de Physico-chimie des aliments, Département de Nutrition, Sciences et Technologie Alimentaires, \\ Faculté des Sciences Agronomiques, Université d'Abomey-Calavi, 01 BP 526, Cotonou, Bénin. \\ 3: Laboratoire d'Écologie Appliquée, Département d'Aménagement et de Gestion des Ressources Naturelles ; \\ Faculté des Sciences Agronomiques, Université d'Abomey-Calavi ; 01 BP 526, Cotonou, Bénin
}

*Auteur correspondant : Prof. Dr. Ir. Paulin AZOKPOTA, Maître de Conférences en Technologie et Microbiologie Alimentaires ; Faculté des Sciences Agronomiques, Université d'Abomey-Calavi, 01 BP 526, Cotonou, Bénin, Email : azokpotap@yahoo.fr ou paulin.azokpota@fsa.uac.bj

Original submitted in on $7^{\text {th }}$ September 2013 Published online at www.m.elewa.org on 30 $30^{\text {th }}$ December 2013. https://dx.doi.org/10.4314/jab.v72i1.99666

\section{RESUME}

Objectif : Le présent travail vise spécifiquement à identifier les espèces de feuilles végétales utilisées traditionnellement comme emballages dans l'artisanat agroalimentaire au sud du Bénin et à décrire les traitements mis en jeu à cet effet.

Méthodologie et résultats : Une enquête sur les emballages-feuilles végétales a été réalisée par ratissage systématique de 150 productrices-vendeuses de denrées alimentaires au sud-Bénin. Les espèces de feuilles végétales ont été identifiées par référence à la base des données disponibles à I'herbier national. Thalia geniculata, (Thalia) Tectona grandis, (Teck) Manihot esculenta (Manioc, manioc doux, cassave), Musa chinensis, (Bananier) Lasimorpha senegalensis (Grand arum du Sénégal, maïs de marécage, oreille d'éléphant) Icacina trichantha (Icacina), Elaeis guineensis (palmier à huile) et Sterculia tragacantha, (Sobou Tagacanthe africain) étaient les principales espèces végétales identifiées et utilisées par environ $98 \%$ des productrices enquêtées. Le rapport pondéral moyen des emballages-feuilles aux denrées alimentaires variait entre 0,05 et $0,53(p / p)$ et pourrait être un indicateur associé à la durée de conservation des aliments.

Conclusion et application : Du fait de leur caractère biodégradable, de leur diversité et d'un éventail de propriétés et caractéristiques intrinsèques, les feuilles végétales utilisées comme emballages jouent un rôle clé dans l'artisanat agroalimentaire au Sud-Bénin. Outre la fonction de protection qu'elles assurent, certaines espèces de feuilles telles que Sterculia tragacantha (Tragacanthe africain), Manihot glaziovii (Manioc caouchouc), Manihot esculenta (Manioc), Thalia geniculata (Thalia) et Tectona grandis (Teck) sont également consommées comme légumes feuilles ou utilisées pour leurs propriétés médicinales pendant que d'autres (Daniellia oliveri (Copalier africain de balsam, santan), Sterculia tragacantha (Sobou, Tragacanthe africain) Canna indica (Canna, Basilier, Grain de plomb de l'Inde), Tectona grandis) transfèreraient leur arome ou couleur aux aliments emballés. Les effets bénéfiques (protecteur, conservateur, nutritionnel, aromatique ou thérapeutique) ou néfastes (toxicité, facteurs 
antinutritionnels) sur les denrées alimentaires emballées doivent être mis en évidence et documentés pour la promotion d'emballages végétaux compatibles avec les normes des emballages modernes et respectueux de l'environnement.

Mots clés Emballages biodégradables, Emballages multi produits, Emballage multi-local ; Biodiversité ; Bénin

\section{Biodiversity of vegetal leaf plants used as food packaging in artisanal food processing in southern Benin.}

Abstract

Objectives: This work aims specifically to identify the species of plant leaves traditionally used as food packaging material in the southern Benin and to describe the required/related treatments.

Methodology and results: A survey was carried out in southern Benin using a systematic sampling of 150 food processors. Identification of plant leaves species was carried out referring to data basis available at the National Herbarium. Thalia geniculata (Thalia), Tectona grandis (Teak), Manihot esculenta (Cassava, Tapioca plant), Musa chinensis (Sweet banana) Lasimorpha senegalensis Lasimorpha senegalensis (senegal big arum I, swampy maize, elephant ear), Icacina trichantha (Icacina plant), Elaeis guineensis (Oil palm) and Sterculia tragacantha (African tragacant)were the main species identified which were used as food packaging material by nearly $98 \%$ of the investigated food processors. The average mass-ratio of plants leaf to food ranged between 0.05 and $0.53(\mathrm{p} / \mathrm{p})$ and could be used as an indicator associated with storage duration of packaged foods.

Conclusions and application of findings: Plant leaves used as packaging material play key role in the food processing in Benin due to their biodegradability, their diversity and the large range of intrinsic proprieties. Except protecting food, some species such as Sterculia tragacantha (Sobou, African tragacant), Manihot glaziovii (Ceara rubber tree), Manihot esculenta (Cassava, Tapioca plant), Thalia geniculata (Thalia) and Tectona grandis (Teak) are also consumed as leafy vegetables or used for their medicinal properties whereas Daniellia oliveri (West African copal, African copaiba Balsam, Sterculia tragacantha (Sobou, African tragacant), Canna indica (wild canna Indian shot), Tectona grandis (Teak) would transfer their aroma and color to the packaged foods. The impact of identified leaves on foods should be investigated in order to point out their positive (aromatic or therapeutic protective, conservative, nutritional,) or negative (toxicity, anti-nutritional factors) effects which can serve as a basis for promoting packaging plant compatible with the standards of modern packaging in the respect of the environmental requirements.

Keywords: Biodegradable packaging; Multiproduct packaging; Multilocal packaging; Benin, Biodiversity

\section{INTRODUCTION}

L'emballage d'un produit est indispensable pour sa protection, son transport, sa promotion et son utilisation (Palling, 1980 ; Paine, 1987). Les matériaux utilisés comme emballages pour les produits alimentaires incluent un certain nombre d'articles (verre, plastique, métal.) dont les feuilles végétales. Ainsi, les feuilles d'un grand nombre d'espèces végétales (Tectona grandis, Musa paradisiaca, Thaumatococcus daniellii etc.) ont pendant longtemps servi à emballer les aliments semi-solides ou solides destinés à être conservés quelques heures, voire quelques jours dans toutes les régions tropicales (Bureau and Tissot, 1987 ; Bureau and Tissot, 1989 ; Adegunloye et al., 2006). En Afrique, ces pratiques longtemps établies étaient essentiellement des moyens physiques de protection des aliments contre les influences externes telles que les microorganismes, l'air, I'humidité (Doyon, 1990). De plus, il a été reconnu que certaines feuilles contiennent des composés actifs - aromatiques, colorants, enzymes (papaïne par exemple), agents antimicrobiens (huiles essentielles) - qui migrent de la feuille végétale vers le produit alimentaire (Buquet and Manchon, 1972 ; Bramsnaes, 1981). En Afrique, des travaux ont répertorié et caractérisé un grand nombre de ces espèces végétales (Hounhouigan, 2000, Adegunloye et al., 2006 ; Adejumo and Ola, 2008) ainsi que les 
risques sanitaires liés à leur usage (Adegunloye et al., 2006, Adejumo and Ola, 2008 ; Crosby, 1981). Par ailleurs, dans le secteur agroalimentaire, les emballages plastiques occupent une position dominante, du fait de leur caractère pratique et esthétique (Pixa, 1988 ; Jenkins and Harrington, 1991). Le Bénin est constamment approvisionné en emballages plastiques en provenance des pays asiatiques, du Nigéria et du Ghana où l'offre est abondante et moins chère. Malgré la prolifération sans cesse croissante des emballages plastiques, il est observé de nos jours, sur presque tous les marchés ruraux et urbains du Bénin, des denrées alimentaires emballées dans les feuilles végétales (Photo 1).

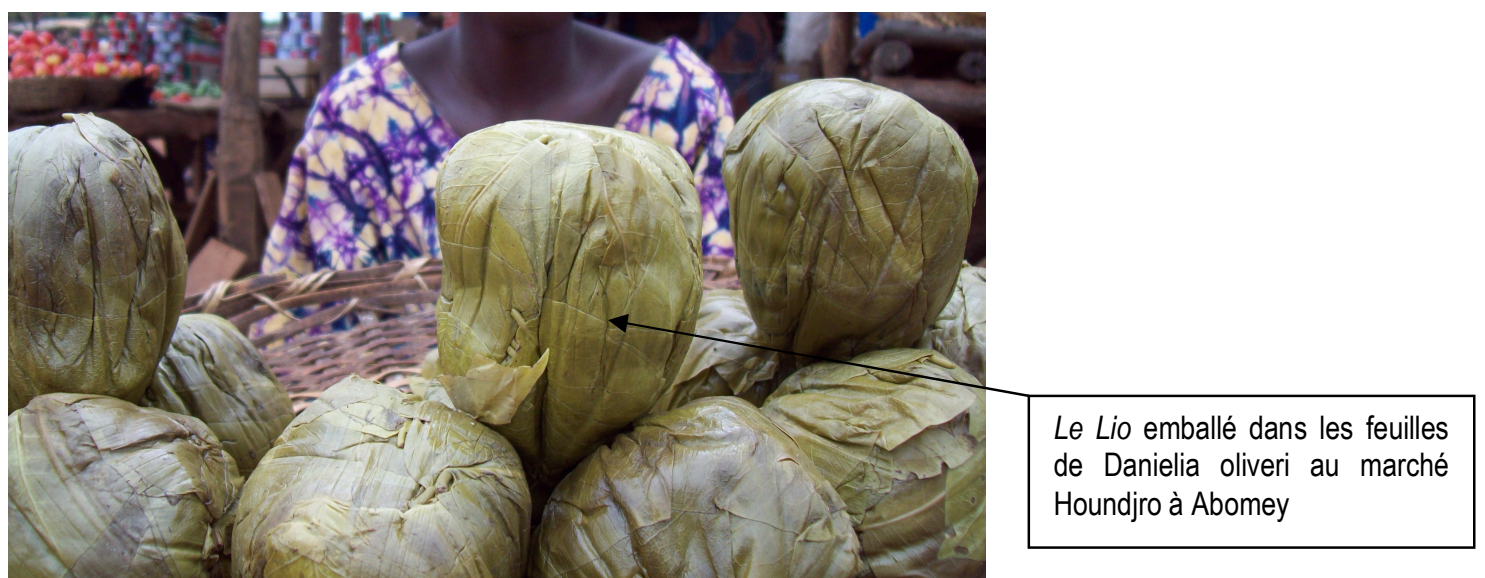

Photo 1 : Le lio, une denrée alimentaire locale emballée dans les feuilles de Danielia oliveri

L'attrait à ce type d'emballage est de plus en plus marqué pour une frange importante de la population qui estime que les emballages plastiques entraînent une contamination chimique du produit emballé, préjudiciable à la santé du consommateur (Dunkl and Steverson, 1987). De plus, du fait de leur caractère non biodégradable, les emballages plastiques posent des problèmes de plusieurs ordres dont la pollution environnementale (insalubrité des villes, bourrage des caniveaux). Dans ces conditions, la promotion des emballages naturels et biodégradables déjà utilisés comme emballagesfeuilles végétales pourrait constituer une piste pour la réduction des impacts environnementaux des emballages plastiques. Un diagnostic rapide de l'offre en emballages végétaux a révélé qu'il existe au Bénin un potentiel important d'emballages alimentaires naturels qui constitue une source importante de revenus, notamment aux femmes (Codjia, 2000 ; Hounhouigan,

\section{MÉTHODOLOGIE}

Échantillonnage et collecte des données : Un sondage sommaire préalable à l'enquête a été réalisé par observation directe sur les marchés et a consisté à recenser les principaux produits de
2000). Cependant, les connaissances endogènes sur les usages et prétraitements des feuilles végétales utilisées comme emballages au Bénin sont peu documentées. A notre connaissance, très peu de travaux ont été réalisés sur ces emballages au Bénin, en particulier les études ethnobotaniques non ciblées ou réalisées dans un espace relativement très restreint (Hounhouigan, 2000). Par ailleurs, les informations sur les types de denrées alimentaires exclusivement emballées dans les feuilles végétales sont loin d'être complètement collectées et documentées. Dans la perspective d'une meilleure valorisation des emballages végétaux au Bénin, le présent travail vise à (i) répertorier les feuilles végétales utilisées comme emballages traditionnels au Bénin et les denrées alimentaires pour lesquelles elles sont utilisées et (ii) décrire les savoir-faire traditionnels utilisés pour le traitement et la confection desdits emballages.

consommation courante pour lesquels les feuilles végétales sont utilisées comme emballage. Les marchés urbains où les feuilles végétales sont fréquemment utilisées comme emballages 
alimentaires ont été retenus. L'enquête proprement dite a été réalisée dans quatre principales zones au sud du Bénin (Abomey-Bohicon, Lokossa, Ouidah et Porto-Novo).Dans chaque localité, 30 productricesvendeuses de denrées alimentaires ont été sélectionnées par la méthode de marche en avant, couplée à celle de la bouteille "Random walk " suivant un ratissage systématique. L'enquête a été réalisée à l'aide d'un questionnaire qui a intégré essentiellement les informations relatives aux espèces végétales utilisées comme emballage alimentaire (leurs appellations locales), à la période de disponibilité, aux denrées alimentaires traditionnellement emballées, aux facteurs influençant leur utilisation et les prétraitements avant

\section{RÉSULTATS ET DISCUSSION}

Profil des groupes socio-culturels enquêtés : Les emballages-feuilles végétales sont utilisés par tous les groupes socio-culturels mais à différents degrés. Les productrices enquêtées étaient issues d'une dizaine de groupes socio-culturels exerçant dans l'agroalimentaire (Tableau 1). Les productrices Fon étaient majoritaires à Abomey $(93,4 \%)$, Bohicon $(100 \%)$, Ouidah $(90 \%)$ tandis que les Kotafon $(83,3 \%)$ et les Goun $(66,7)$ étaient les plus nombreux, respectivement, à Lokossa et à Porto-Novo. Les groupes socio-culturels minoritaires Idatcha, Mahi, usage. Par ailleurs, le nombre de feuilles et de couches par produit emballé, le poids de l'emballage et du produit contenu ont été déterminés au laboratoire.

Traitement des données collectées : Les informations recueillies ont été complétées par l'identification taxonomique des espèces en se servant des guides (Akoègninou et al., 2006 ; de Souza, 1987 ; de Souza, 1988 et de Souza, 2006). L'identification taxonomique a été confirmée au laboratoire de l'Herbier National du Bénin en utilisant la base des données disponible à cet effet. Les données collectées ont été soumises à la statistique descriptive au moyen de l'Excel.

Holi, Saxwè, Tori et Séto ont été également identifiés dans les localités enquêtées, comme utilisateurs traditionnels des feuilles végétales comme emballage des produits alimentaires. La plupart des productrices enquêtées (95\%) étaient dans une tranche d'âge comprise entre 30 et 55 ans. Toutes les productrices enquêtées ont affirmé avoir acquis suffisamment d'expérience d'au moins 5 à 30 années pour l'utilisation des emballages végétaux dans le domaine de la transformation agro-alimentaire.

Tableau 1 : Répartition des enquêtés utilisateurs d'emballages feuilles végétales par localité $(\mathrm{N}=30)$ et groupe socio-culturel

\begin{tabular}{ccc}
\hline Localité & Groupe socio-culturel & (\%, répondants) \\
\hline \multirow{2}{*}{ Abomey (AB) } & Fon & 93,4 \\
& & 3,3 \\
& Idatcha & 3,3 \\
& Mahi & 3,3 \\
\hline Bohicon (BC) & Fon & 100 \\
\hline \multirow{2}{*}{ Lokossa (LK) } & Kotafon & 83,3 \\
& Fon & 13,3 \\
& Saxwè & 3,4 \\
\hline \multirow{3}{*}{ Ouidah (OD) } & Fon & 90 \\
& Holi & 6,7 \\
& Goun & 3,3 \\
\hline \multirow{3}{*}{ Porto- Novo (PN) } & Goun & 66,7 \\
& Sèto & 16,7 \\
& Tori & 10 \\
& Fon & 6,7 \\
\hline
\end{tabular}

Principales espèces végétales identifiées comme emballages des produits alimentaires : Les espèces végétales dont les feuilles sont utilisées comme emballages des produits alimentaires ainsi que les produits associés sont présentées dans le tableau 2. Au total, une trentaine (28) d'espèces végétales a été identifiée comme emballages des produits, essentiellement, à base de céréales, de légumineuses ou d'oléagineux (Tableau 2). Certaines espèces végétales telles que Musa sapientum, Musa 
chinensis, Thalia geniculata, Tectona grandis ont été identifiées dans toutes les localités enquêtées et servent d'emballage de divers produits alimentaires, notamment le Ogui, le Ablo, le Kandji, le Aboété, le
Kpo, le Adjagbé, le Abla, le Gowé, et le Abotin (Hounhouigan et al., 1998). On peut les désigner comme espèces végétales emballages multiproduits (Tableau 2).

Tableau 2 : Diversité des emballages feuilles et denrées alimentaires emballés par localité

\begin{tabular}{|c|c|c|c|c|c|c|c|}
\hline Espèces végétales & Noms locaux & Denrées alimentaires & $\begin{array}{r}\text { Pource } \\
\text { loca }\end{array}$ & $\begin{array}{l}\text { tage }(\% \\
\text { ité }\end{array}$ & de ré & ondant & \\
\hline & & & $A B$ & $\mathrm{BC}$ & LK & OD & PN \\
\hline Daniellia oliveri & Zaman $^{1}$ & Lio & 30 & 30 & - & - & - \\
\hline Manihot glaziovii & Loba man ${ }^{1}$ & Lio & 30 & 30 & - & - & - \\
\hline Manihot esculenta & Finyin $\operatorname{man}^{1}$ & Lio & 30 & 30 & 50 & 50 & \\
\hline $\begin{array}{l}\text { Calopogonium } \\
\text { mucunoides }\end{array}$ & Akpaman 1 & Lio & 10 & 16,66 & - & - & - \\
\hline Ficus capensis & Voman ${ }^{1}$ & Lio & - & - & 10,66 & 6,66 & - \\
\hline Blighia sapida & Lissè man ${ }^{1}$ & Lio & 3,33 & 6,66 & - & - & - \\
\hline Sterculia tragacantha & Hongbèdè man ${ }^{1}$ & Abla, Ogui & & & 40 & 23,33 & 23,33 \\
\hline Pouteria alnifolia & $\begin{array}{l}\text { Plokissa man }{ }^{1} \\
\text { Funfun man }{ }^{2} \\
\text { Foufouin } \text { man }^{3}\end{array}$ & Ogui & - & 10 & - & 20 & 60 \\
\hline Musa sapientum & Kokoéman ${ }^{1}$ & $\begin{array}{c}\text { Ogui, ablo,kandji, aboété, } \\
\text { kpo,adjagbé, } \\
\text { abla,kowé,abloyoki }\end{array}$ & 13,33 & 16,66 & 66,66 & 33,33 & 66,66 \\
\hline Tectona grandis & Xwlèman ${ }^{1}$ & $\begin{array}{l}\text { Ogui, abla, gowé, } \\
\text { abotin, afitin, aboété }\end{array}$ & 40 & 60 & 40 & 66,66 & 80 \\
\hline Holarrhena floribunda & Lètinman 1 & Lio & 23,33 & 20 & - & - & - \\
\hline Thalia geniculata & Afléman'1 & Lio, Ogui ,gowé, abotin & 10 & 16,66 & 13,33 & 46,66 & 83,33 \\
\hline $\begin{array}{l}\text { Lasiomorpha } \\
\text { senegalensis }\end{array}$ & Toungoman ${ }^{1}$ & Lio, Ogui, kandji & -- & - & - & 36,66 & 50 \\
\hline Cussonia arborea & Toflo gotun 1 & Lio & - & - & - & - & 16,66 \\
\hline Isoberlinia doka & Kpakpa man ${ }^{1}$ & Lio & 10 & 10 & - & - & - \\
\hline Gmelina arborea & Fofitin man ${ }^{1}$ & Lio & - & 10 & - & - & - \\
\hline Ficus embellata & Voliman 1 & Lio & - & - & 13,33 & - & - \\
\hline Lannea microcarpa & Zuzu man ${ }^{1}$ & Lio & - & 13,33 & - & - & - \\
\hline Musa chinensis & Avlan man3,4 & Lio, Ogui & 30 & 20 & 66,66 & 33,33 & 66,66 \\
\hline $\begin{array}{l}\text { Canna indica } \\
\text { Triplochiton } \\
\text { scleroxylon }\end{array}$ & $\begin{array}{l}\text { Tiblikoun man }{ }^{3} \\
\text { Atiwé }^{3}\end{array}$ & $\begin{array}{l}\text { Lio, ogui } \\
\text { Lio }\end{array}$ & - & - & $\begin{array}{c}33,33 \\
30\end{array}$ & - & - \\
\hline Antiaris toxicaria & Guxoman 3 & Lio & & & & 3,33 & - \\
\hline Anthocleista vogelii & Gotun 1 & Lio & & & & 13,33 & 16,66 \\
\hline Icacina trichantha & Agbégbéman ${ }^{4}$ & Ogui ,abloyoki & - & - & - & - & 76,66 \\
\hline $\begin{array}{l}\text { Polygonum } \\
\text { senegalense }\end{array}$ & Towé4 & Ogui & - & - & - & - & 23,33 \\
\hline $\begin{array}{l}\text { Sarcocephalus } \\
\text { latifolius }\end{array}$ & Koman ${ }^{1}$ & Ogui & - & - & - & 3,33 & - \\
\hline Elaeis guineensis & Déman1 & Lio, afitin & 56,66 & 63,33 & 50 & 50 & - \\
\hline
\end{tabular}

${ }^{*}$ AB (Abomey): BC (Bohicon); LK (Lokossa); OD (Ouidah); PN (Porto-Novo)). 1: Groupe socio-culturel Fon ; 2: Groupe socio-culturel Saxwè ; 3 : Groupe socio-cultutel Kotafon ; ${ }^{4}$ : Groupe socio-culturel Goun ; * : Denrée Alimentaires détaillées dans le tableau 3

Parmi les espèces collectées, plusieurs servent à l'emballage d'un type de produit et vice versa. De plus, on observe la tendance à une territorisation ou adaptation de l'usage aux espèces végétales 
emballages. En effet, les espèces végétales Daniellia oliveri, Manihot glaziovii, Calopogonium mucunoides, Blighia sapida, Holarrhena floribunda, Gmelina arborea, Isoberlinia doka et Lannea microcarpa étaient majoritairement utilisées par les productrices pour emballer le Lio (pâte de maïs) à Abomey ou Bohicon, tandis qu'à Lokossa et à Ouidah, Ficus capensis, Antiaris toxicaria ssp. welwitschii et Sarcocephalus latifolius étaient les espèces végétales identifiées servant d'emballage pour le Lio. Ces feuilles apparaissent comme des espèces végétales-emballages à envergure zonale/locale. En outre, l'espèce Musa sapientum, par exemple, servait, à lui seul, à emballer plusieurs produits tels Ogui (maïs), ablo (maïs et riz), kandji (maïs), aboété (maïs), kpo (maïs), adjagbé (Légume+ maïs), abla (niébét maïs), kowé (maïs +niébé), abloyoki (racines). On peut les désigner comme espèces végétales emballages multiproduits (Tableau 2). L'adaptation/territorisation des usages est probablement guidée par la préférence des consommateurs à un type d'emballage donné ou par la disponibilité des emballages dans la localité, elle même liée à l'écosystème spécifique de la localité. En effet, certaines espèces telles que Thalia geniculata et Polygonum senegalense ne poussent que dans les zones marécageuses souvent rencontrées à Porto-Novo ou Lokossa. D'autres espèces ne peuvent se développer que sur la terre ferme dans les plateaux (Abomey-Bohicon). Cependant, la plupart des emballages se rencontrent dans presque toutes les localités par le biais des activités commerciales favorisant leur transfert des campagnes vers les villes ou d'une ville à une autre. C'est en partie ce qui justifie que certaines espèces (Tectona grandis, Thalia geniculata, Musa sapientum etc.) soient à la fois des emballages multilocales et multiproduits. Des travaux antérieurs avaient déjà mentionné l'usage de certaines espèces comme emballages des produits alimentaires. En effet, Adegunloye et al. (2006) avaient étudié l'influence des feuilles-emballages de Thaumatococcus daniellii, Musa paradisiaca, Tectona grandis sur la qualité microbiologique des aliments pendant que Adejumo and Ola (2008) ont identifié Thespesia populnea, Marantochloa spp et plantain (Musa chinensis) et les spaths de maïs (Zea mays) comme espèces végétales communément utilisées comme emballages des denrées alimentaires au Nigéria. Cependant, le nombre d'espèces reste limité au regard de la diversité observée dans notre collecte.
Par ailleurs, les caractéristiques sensorielles des feuilles emballages peuvent influencer leur usage "zonal, multiproduit ou multilocal ». En exemple, les feuilles des espèces Thalia geniculata et Daniellia oliveri, particulièrement reconnues, pour leur arôme et leur effet conservateur sont utilisées pour l'emballage de Lio à Abomey - Bohicon. De même, les feuilles de Sterculia tragacantha sont préférées par les productrices de Ouidah pour emballer le Abla, tandis que les feuilles de Icacina trichantha servent d'emballage pour le Ogui par les productrices de Porto-Novo (Nago, 1997, Nago, 1998). Ce rôle conservateur et/ou sensoriel des feuilles végétales emballages des produits alimentaires avait déjà été rapporté par Buquet and Manchon (1972) et Bramsnaes (1981) en liaison avec leur composition en éléments actifs-aromatiques ou colorants qui migreraient de la feuille vers le produit alimentaire au cours de la conservation après l'emballage. Cependant, des contaminations microbiologiques ont été observées au niveau des denrées emballées dans les feuilles végétales après 2 à 4 jours de conservation (Adegunloye et al., 2006, Adejumo and Ola, 2008).

Variété des produits alimentaires emballés dans les feuilles végétales : Les aliments emballés dans les feuilles végétales varient en état (frais, chaud, sémi-liquide, pâteux, solide) et en groupes alimentaires. En ce qui concerne les denrées alimentaires, trois principaux groupes de produits alimentaires emballés dans les feuilles végétales ont été recensés : céréales, légumineuses et racines. Les produits à base de céréales tels que le Lio, le Ogui, le Kandji, le Gowé, le Abotin, le Ablo, le Com et le Kpo (Nago et Hounhouigan, 1998a, Nago et Hounhouigan, 1998b ; Vieira-Dalodé et al., 2007) sont des denrées alimentaires, pour la plupart, retrouvées dans toutes les localités enquêtées (Tableau 3). Ils sont emballés soit, à chaud après cuisson (Ogui, Gowé), soit à froid avant cuisson (Com, Kandji, Ablo etc.). Par rapport aux aliments à base de légumineuses, le Afitin, le Abla sont les plus représentatifs. Ils sont tous emballés à froid à la température ambiante $\left(25-30^{\circ} \mathrm{C}\right)$, l'un sans cuisson (Afitin), l'autre suivi de cuisson à vapeur (Hounhouigan et al, 1998 ; Azokpota et al., 2006). Le troisième groupe concerne les produits dérivés des racines et tubercules tel que l'Abloyoki obtenu du manioc. Ce dernier est emballé avant cuisson au four traditionnel (Nago et Hounhouigan, 1998b). 
Tableau 3 : Principales denrées alimentaires emballées dans des feuilles végétales

\begin{tabular}{|c|c|c|c|c|c|c|}
\hline \multirow{2}{*}{$\begin{array}{l}\text { Denrées } \\
\text { alimentaires }\end{array}$} & \multirow[t]{2}{*}{ Description } & \multicolumn{5}{|c|}{ Lieux d'identification } \\
\hline & & $A B$ & $\mathrm{BC}$ & LK & OD & PN \\
\hline Lio $^{1}$ (céréales) & $\begin{array}{l}\text { Pâte fermentée de maïs, consistante et compacte, obtenue } \\
\text { par cuisson à la vapeur, emballée avant d'être cuite }\end{array}$ & + & + & + & + & - \\
\hline Ablo ${ }^{1}$ (céréales) & $\begin{array}{l}\text { Pâte fermentée à la levure, légèrement salée et sucrée, } \\
\text { obtenue par cuisson à la vapeur, préparée à partir d'une } \\
\text { bouillie avec du mawè à laquelle est ajoutée de la farine de } \\
\text { maïs, de blé ou du riz; emballée, avant d'être cuite à la } \\
\text { vapeur. }\end{array}$ & + & + & + & + & - \\
\hline $\begin{array}{l}\text { Gowé, Abotin } 1 \\
\text { (céréales) }\end{array}$ & $\begin{array}{l}\text { Pâte légèrement sucrée, préparée à partir du maïs ou du } \\
\text { sorgho germé et fermenté ; consommé sous forme de } \\
\text { bouillie glacée désaltérante après reconstitution dans l'eau }\end{array}$ & + & + & - & - & - \\
\hline Kandji1 (céréales) & $\begin{array}{l}\text { Pain traditionnel préparé à partir de la farine; emballé avant } \\
\text { d'être cuite au four. }\end{array}$ & + & + & - & + & - \\
\hline $\begin{array}{l}\text { Aboété } 1 \\
\text { (céréales) }\end{array}$ & $\begin{array}{l}\text { Pâte préparée à partir du maïs frais et des ingrédients (Huile, } \\
\text { piment, sel, niébé); ; emballé avant d'être cuit à la vapeur. }\end{array}$ & + & + & - & - & - \\
\hline Com (céréales) & Pâte fermentée à partir du maïs ; emballé avant d'être cuite & - & - & ++ & - & - \\
\hline $\mathrm{Kpo}^{1}$ (céréales) & $\begin{array}{l}\text { Pâte de maïs assaisonnée de piment, d'oignon, de sel et } \\
\text { d'ail ; emballée avant d'être cuite à la vapeur. }\end{array}$ & + & + & - & - & - \\
\hline $\begin{array}{l}\text { Adjagbé } 1 \\
\text { (céréales) }\end{array}$ & $\begin{array}{l}\text { Pâte de farine de maïs mélangée avec des feuilles de niébé } \\
\text { et assaisonné : emballée avant d'être cuite à la vapeur. }\end{array}$ & + & + & - & - & - \\
\hline $\begin{array}{l}\text { Abla }^{1} \text { (céréales+ } \\
\text { légumineuse) }\end{array}$ & $\begin{array}{l}\text { Pâte préparée à partir d'un mélange de maïs concassé et de } \\
\text { niébé ; emballée avant d'être cuite à la vapeur. }\end{array}$ & + & + & - & + & - \\
\hline $\begin{array}{l}\text { Kowé }{ }^{1} \text { (céréales+ } \\
\text { légumineuse) }\end{array}$ & $\begin{array}{l}\text { Pâte de farine de maïs et de gritz de niébé mélangée avec } \\
\text { du jus de noix de palme. Ce produit est également emballé } \\
\text { dans les feuilles végétales avant d'être cuit à la vapeur. }\end{array}$ & + & + & - & - & - \\
\hline $\begin{array}{l}\text { Abloyoki } \\
\text { (racines) }\end{array}$ & $\begin{array}{l}\text { Pain traditionnel à base de manioc râpé, cuit au four emballé } \\
\text { avant d'être séché }\end{array}$ & - & - & - & - & + \\
\hline $\begin{array}{l}\text { Ogui }{ }^{1} \text { (céréales) } \\
\text { Kannan }^{4} \\
\text { Akansan } \\
\text { Afitin } \\
\text { (légumineuses) }\end{array}$ & $\begin{array}{l}\text { Pâtes de maïs issues de fermentation acide, obtenues par } \\
\text { cuisson du culot, après addition d'eau ou du surnageant, } \\
\text { emballé après cuisson } \\
\text { Condiment, obtenu à partir de la fermentation alcaline des } \\
\text { graines de néré (Parkia biglobosa) ; mis en boule et emballé } \\
\text { enroulées des folioles de palmier après fermentation }\end{array}$ & + & + & + & + & ++ \\
\hline
\end{tabular}


Contribution spécifique des emballages feuilles au poids unitaire des denrées alimentaires : Le poids moyen des emballages feuilles par unité de produit varie entre $3,65 \mathrm{~g}$ et $81,97 \mathrm{~g}$, respectivement pour l'lcacina trichantha (cas abloyoki) et Pouteria alnifolia (cas du lio). Le rapport massique (masse emballage/masse produit) ou rapport pondéral feuilleproduit renseigne sur la contribution/part des feuilles dans le poids des produis emballés. Ce rapport variait entre 0,05 et 0,53 , avec Kandji emballé dans Lasimorpha senegalensis donnant la plus forte valeur et abloyoki emballé dans l'Icacina trichantha la plus faible valeur (Tableau 4). Cette variation semble beaucoup plus liée au type de produit emballé qu'aux emballages feuilles utilisés. En effet, pour le ogui, le rapport massique est de 0,11 lorsqu'il est emballé dans Thalia geniculata et de 0,13 quand il est emballé dans Tectona grandis. De même, le rapport massique des emballages pour le Kandji et le Ogui, deux pâtes obtenues par cuisson étaient de 0,53 et 0,11-0,13, respectivement (Tableau 4). II est possible que ce paramètre soit l'indicateur le plus pertinent directement lié à la durée de conservation, étant entendu que le kandji et le ablo, présentant des rapports massiques élevés (respectivement, 0,53 et 0,38 ) se conservent mieux (environ 5 à 7 jours). Par ailleurs, certaines feuilles végétales emballages sont également utilisées à des fins médicinales. Les feuilles de manioc (Manihot esculenta), et les jeunes feuilles de teck (Tectona grandis) mélangés au lait cru avec ajout du sucre constitueraient une recette contre l'anémie. De même, les feuilles de Thalia geniculata cuites dans le surnageant de pâte fermentée de maïs (Ogui) seraient une tisane contre le paludisme. Les feuilles séchées de Tectona grandis cuites dans de l'eau sous forme de tisane servent d'antibiotique pour la toilette des enfants. Toutefois, en dépit des faits quotidiens probants, toutes ces informations sur les utilisations médicinales proviennent des témoignages et des sources, transmis oralement (notamment par les utilisatrices des emballes végétaux) et ne sont pas encore soutenues par des données régulièrement documentées. Au plan alimentaire, les feuilles de Sterculia tragacantha ou Triplochiton scleroxylon sont utilisées comme légume dans la préparation des sauces

gluantes 
Tableau 4: Poids unitaire des produits et rapport massique des espèces de feuilles végétales utilisées comme emballage alimentaire.

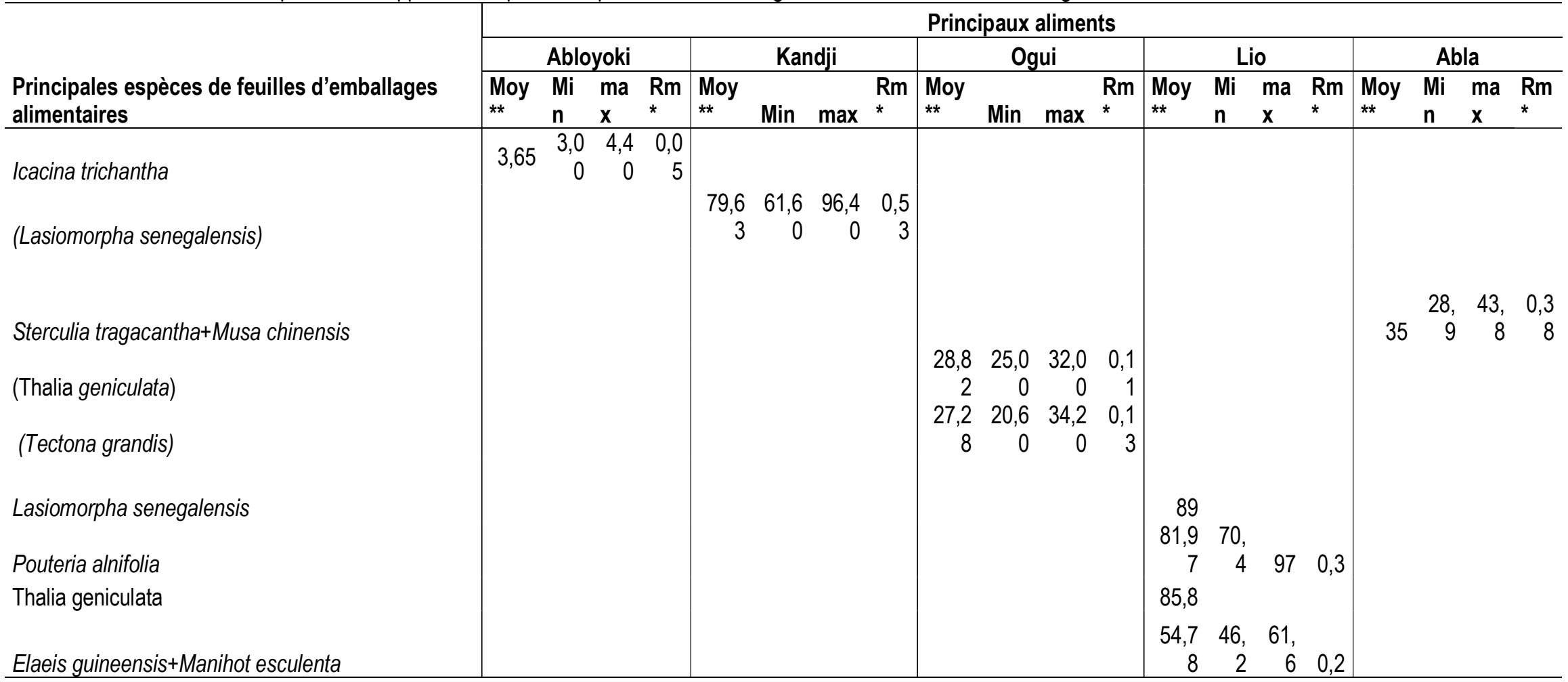

*: $\mathrm{Rm}=$ rapport massique: masse emballage divisée par masse produit (masse emballage/masse produit)

**:Poids de l'emballage par unité de produit, moyenne de 6 observations (g) 
Prétraitements des feuilles végétales emballages avant usage : Les prétraitements appliqués aux emballages feuilles-végétales dépendent de la période de cueillette, de la nature de la feuille ou du produit alimentaire à emballer. En effet, pour les feuilles disponibles en saison des pluies, un simple nettoyage est suffisant car selon les productrices, ces feuilles seraient moins sales. C'est le cas des feuilles de Manihot esculenta, Manihot glaziovii et de Tectona grandis qui sont abondantes en saison pluvieuse. Par contre, en saison sèche, le lavage à eau est généralement appliqué aux feuilles destinées à l'emballage car elles sont très poussiéreuses. Le flambage, par exemple, est une opération qui réduirait la charge microbienne à la surface des feuilles de Musa sapientum en même temps qu'elle permet de les ramollir pour les rendre plus malléables. Les feuilles de Thalia geniculata, Holarrhena floribunda, Canna indica, Lasimorpha senegalensis subissent particulièrement un blanchiment pour devenir plus résistantes au déchirement et plus malléables. Ces différents prétraitements sont probablement inappropriés pour assurer une sécurité sanitaire aux denrées emballées comme l'ont constaté Adejumo et Ola (2008) qui ont rapporté un important développement de moisissures dans le Agidi, un produit d'origine nigériane après 4 jours de conservation dans les feuilles végétales. Par ailleurs, certaines feuilles sont parfois conservées trempées dans l'eau quelques jours (24-72 heures) avant les prétraitements. D'autres, telles que les feuilles de Tectona grandis peuvent se conserver pendant une semaine si elles sont bien rangées dans des sacs de jute avec leurs extrémités bien attachées. Une autre technique de conservation consiste à tapisser les feuilles dans un panier et à les recouvrir de tissus pour éviter leur assèchement sous l'effet direct des rayons solaires.

Techniques de confection des emballages végétaux : Les techniques de confection des emballages végétaux sont les mêmes dans toutes les localités enquêtées. Cependant, elles varient en fonction de la nature du produit alimentaire (semiliquide ou solide) ou du mode d'obtention du produit (emballage avant ou après préparation). Certaines denrées alimentaires (Ogui) sont emballées à chaud au cours de la préparation, notamment au stade de produit semi-fini après préparation, certains, juste avant la cuisson (Abloyoki), d'autres enfin, à température ambiante (Afitin). Des pratiques similaires ont été rapportées au Nigéria (Adejumo et Ola, 2008). En général, deux couches de feuilles sont utilisées par produit emballé : la première couche de feuilles (2-4 unités) est interne et est directement en contact avec le produit. Cette couche de feuilles subit un prétraitement (flambage ou blanchiment). La deuxième couche est externe ; elle sert simplement d'enveloppe de protection, avec un prétraitement facultatif. La technique de confection des emballages pour le Lio requiert un savoir-faire particulier car il s'agit d'un véritable habillement du produit, avec le rapport pondéral feuilles-produit variant entre $1 / 3$ à $1 / 2(p / p)$. En effet, les feuilles sont d'abord équeutées et tapissées par lot d'une dizaine environ. Ensuite, un creux est formé à l'intérieur des feuilles tapissées où le lio à emballer est introduit. L'ensemble, ajusté et fermé, est attaché avec des folioles de branches de palmier dans un double enroulement (Photo 2).

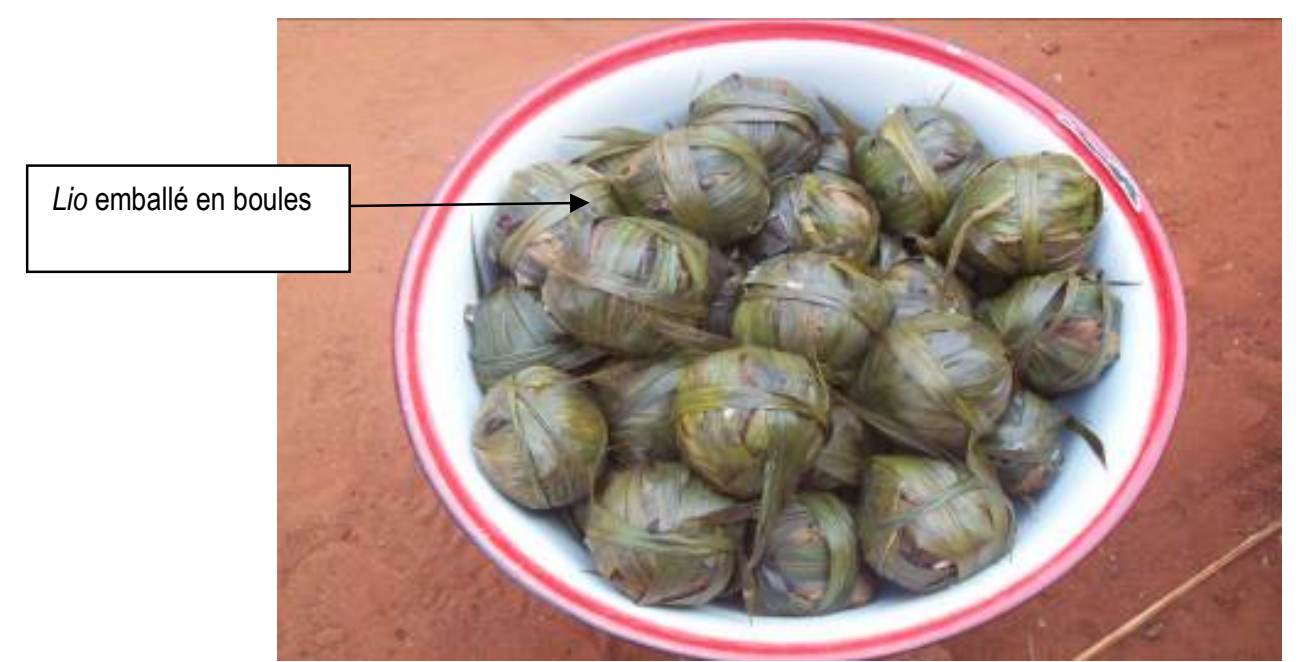

Photo 2 : Le lio emballé dans les feuilles de Manihot esculenta et enroulé de folioles de palme (Elaeis guineensis) 


\section{Onzo et al. J. Appl. Biosci. 2013. Biodiversité des emballages-feuilles végétales utilisées dans l'artisanat agroalimentaire , Benin}

Les feuilles qui subissent cette technique proviennent essentiellement des espèces végétales Manihot esculenta, Manihot glaziovii, Holarrhena floribunda, Gmelina arborea et Isoberlinia doka. Une variante est utilisée pour emballer le Ogui. Elle consiste à réaliser une pile de feuilles qui emballent/enveloppent le produit (Photo 3). Les feuilles utilisées pour cette variante proviennent des espèces végétales Tectona grandis, Pouteria alnifolia, Thalia geniculata, Musa sapientum et Lasiomorpha senegalensis.

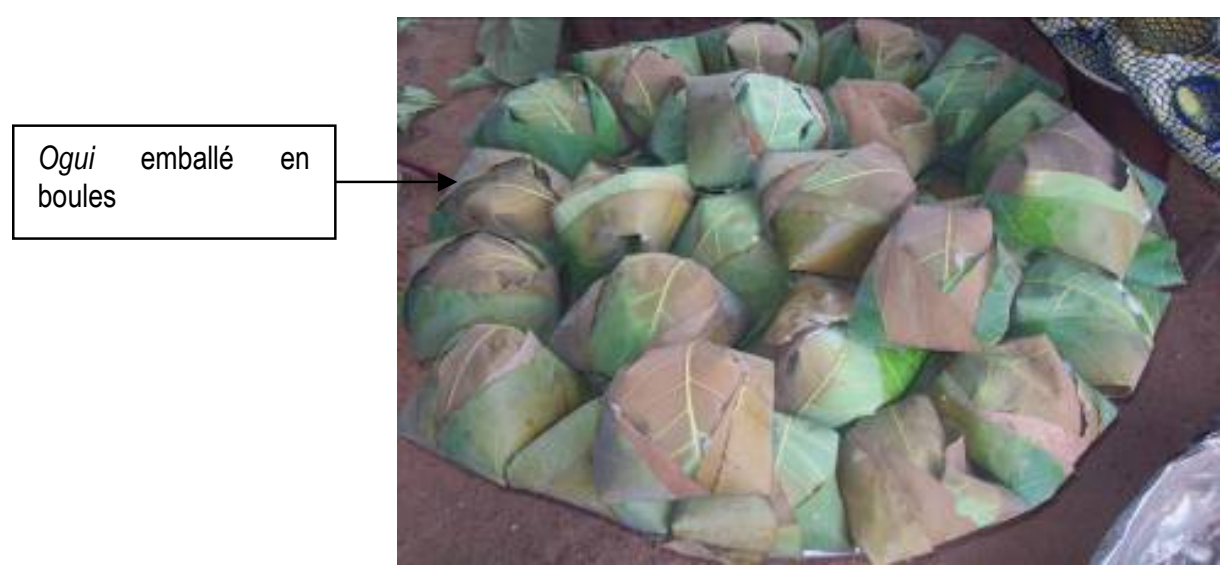

Photo 3 : Ogui emballé dans des feuilles de Isoberlinia doka

L'ensemble de ces savoir-faire devra être mieux utilisé dans la perspective de mieux valoriser les emballages-feuilles utilisés dans l'artisanat

\section{CONCLUSION}

Au regard de la gamme des denrées alimentaires emballées, les espèces végétales dont les feuilles sont utilisées comme emballage méritent une attention particulière au niveau des opérations de prétraitements et de confection. Outre leur rôle de protection des denrées, ces feuilles végétales concèdent aux produits des substances aromatiques, colorantes et parfois conservatrices. Les relations

\section{REMERCIEMENTS}

Nous exprimons notre profonde gratitude à l'Union Économique Monétaire Ouest Africaine (UEMOA) qui a financé le présent travail dans le cadre du

\section{RÉFÉRENCES BIBLIOGRAPHIQUES}

Adegunloye DV, Agarry OO, Adebolu TT, Adetuyi FC, 2006. Effect of leaf-packaging on the microbiological assessment of some food items. African Journal of Biotechnology Vol. 5 (5): 445-447.

Adejumo BA, Ola FA, 2008. The appraisal of local food packaging materials in Nigeria. Continental J. Engineering Sciences 3:1320.

Akoègninou $A$, Van der Burg, Van der Maesen $W \mathrm{~J}$, Adjakidjè $V$, Essou $J \mathrm{P}$, Sinsin $\mathrm{B}$, Yédomanhan $\mathrm{H}, 2006$. Flore analytique du agroalimentaire, non seulement au Bénin, mais également dans la sous-région où la pratique demeure encore très vivace.

entre le mode de traitement et le statut sanitaire (contamination microbienne et chimique), la composition physico-chimique des espèces végétales étudiées ou les échanges entre le produit et la feuille ou avec le milieu externe doivent être déterminés au cours des travaux ultérieurs pour une bonne compréhension de leur effet conservateur et/ou aromatique.

Programme PAES (Programme d'Appui à l'Enseignement Supérieur).

Bénin. Cotonou \&Wageningen. $1034 \mathrm{p}$.

Azokpota P, Hounhouigan DJ, Nago MC, 2006. Microbiological and chemical changes during the fermentation of African locust bean (Parkia biglobosa) to produce afitin, iru and sonru, three traditional condiments produced in Benin. International Journal of Food Microbiology 107: 304-309.

Bramsnaes F, 1981. Maintaining the quality of frozen Foods during distribution. Food Technology 35 (4) : 38-43. 
Buquet A and Manchon P, 1972. Emballages flexible et conditionnement des produits alimentaires. Cahiers de Nutrition et de Diététique 7 (2) : 105-124.

Bureau GP B and Tissot L, 1987. Conditionnement et qualité : critères de choix. Propriétés. Principes généraux et neutralité alimentaire. Viandes et Produits Carnés 8 (1) : 13-15.

Bureau $G$ and Multon J L, 1989. L'emballage des denrées alimentaires de grande consommation. Lavoisier, Paris. 730p.

Codjia RP, 2000. La prolifération des déchets plastiques à Cotonou : Effet sur la santé et sur le développement durable. Mémoire de DEA, UNB. 54p.

Crosby NT, 1981. Food packaging materials. Aspects of analysis and migration of contaminants. Applied Science Publisher, London, Royaume-Uni. 190p.

de Souza, SDE 1987. Flore du Bénin. Catalogue des plantes du Bénin, Tome 1. Edition Notre Dame, Université Nationale du Bénin, Cotonou (Bénin). 87p.

de Souza SDE, 1988. Flore du Bénin. Noms des plantes dans les langues nationales $d u$ Bénin, Tom 3, Edition Notre dame. Université Nationale du Bénin, Cotonou (Bénin). 421p.

de Souza SDE, 2006. Flore du Bénin. Plantes de Bord de mer, de Mangrove et de jardins, Tome 2. Edition Notre Dame, revue et complétée. Université Nationale du Bénin, Cotonou (Bénin). 152p.

Doyon G, 1990. La conservation d'un aliment : les mécanismes de dégradation et le choix d'un emballage. Canadian Institute of Food Science and Technology Journal 23 (4): 165-170.

Dunkl EYWL and Stevenson K.E, 1987. Ultra high temperature processing and aseptic packaging of dairy products. Journal of Dairy Science 70 (10): 2192-2020.

Hounhouigan DJ, Nago CM, Monhouanou J, Egounlety M, 1998. La transformation alimentaire traditionnelle des légumineuses et oléagineux au Bénin. Université d'Abomey-Calavi. Faculté des Sciences Agronomiques. Les Publications du CERNA $3: 121 \mathrm{p}$.

Hounhouigan DJ, 2000. Matières végétales au Bénin. Un potentiel d'emballages biodégradables. In. Bulletin du Réseau TPA : 17. Les emballages alimentaires. 29-41.
Jenkins WA and Harrington JP, 1991. Packaging Foods with plastics. Technomic Publishing Co., Lancaster. 326p.

Nago MC, 1997. La transformation Alimentaire Traditionnelle du maïs au Bénin : Détermination des caractéristiques physicochimiques des variétés en usage ; Relation avec l'obtention et la qualité des principaux produits dérivés ; Doctorat d'Etat EsSciences. 199p.

Nago CM, 1998. Technologies traditionnelles et alimentation au Bénin : Aspects techniques, biochimiques et nutritionnels, Université Nationale du Bénin, Abomey-Calavi, Bénin. $222 \mathrm{p}$.

Nago CM and Hounhouigan DJ, 1998a. La transformation alimentaire traditionnelle des céréales au Bénin. Université d'AbomeyCalavi. Faculté des Sciences Agronomiques. Les Publications du CERNA, 1. 150p.

Nago CM and Hounhouigan DJ, 1998b. La transformation alimentaire traditionnelle des racines et tubercules au Bénin. Université d'Abomey-Calavi. Faculté des Sciences Agronomiques. Les Publications du CERNA 2. $97 \mathrm{p}$

Paine FA, 1987. Modern processing, packaging and distribution systems for foods. Blackie and Son Ltd., Glasgow, Royaume-Uni. 163p.

Palling SJ, 1980. Developments in foods Packaging. Applied Sciences Publishers, Barking, Royaume-Uni. $190 \mathrm{p}$

Pixa R, 1988. L'utilisation de matières plastiques pour l'assemblage des produits alimentaires. Annales des falsifications, de l'expertise chimique et toxicologique 81 (872) : 447450.

Vieira-Dalodé G, Jespersen L, Hounhouigan DJ, Moller PL, Nago CM, Jakobsen M, 2007. Lactic acid bacteria and yeasts associated with gowe production from sorghum in Benin. Journal of Applied Microbiology 103 (2): 342-349. 